(2)

DER LITERARISCHEN Wertung 
Walter Müller-Seidel

\title{
Probleme
}

\section{DER LITERARISCHEN WERTUNG}

\author{
ÜBER DIE \\ WISSENSCHAFTLICHKEIT \\ EINES \\ UnWISSENSChaftlichen Themas
}

J. B. Metzlersche

VERLAGSBUCHHANDLUNG

StUTtgart 
Zweite, durchgesehene Auflage 1969

ISBN 978-3-476-00121-4

ISBN 978-3-476-02978-2 (eBook)

DOI 10.1007/978-3-476-02978-2

(C) 1965 Springer-Verlag GmbH Deutschland

Ursprünglich erschienen bei J. B. Metzlersche Verlagsbuchhandlung und Carl Ernst Poeschel Verlag GmbH in Stuttgart 1965 
Meiner Frau zugeeignet 


\section{INHALT}

\section{VORÜBERLEGUNGEN 1}

Wertungen außerhalb der Literaturwissenschaft. Die Unwissenschaftlichkeit des Themas in Geschichtswissenschaft und Philologie. Das Veralten der Resultate. Max Webers Werturteilsfreiheit. Das Ideal der voraussetzungslosen Wissenschaft. Das Spannungsgefüge der Wissenschaft. Bedenken gegenüber problemloser Wertfreudigkeit. Das Verstehen und die Probleme der Hermeneutik. Die Selbstgefälligkeit der Kritik. Die Vorurteile: Allegorie, Barock, Heine. Indirekte Wertungen. Interpretatorische Ergiebigkeit. Die Übergänge in der Literatur. Die reduzierte Interpretation. Bejahung der literarischen Wertung. Höhere Reflexionsstufen der Fragestellung. Das Beispiel des Klassischen. Wertung als Editionsproblem. Die Literaturgeschichtsschreibung. Revisionen des Wertrelativismus. Bedingungen der literarischen Wertung: das geschichtliche Denken, der Zeitpunkt des Urteils, die Dignität des Urteils.

Der erste Fragenkreis: Die Probleme des Öffentlichen

41

Die Welt- und Völkergabe der Dichtung. Spiel und Offentlichkeit. Die Esoterik. Transzendierungen des Privaten: Thomas Manns ,Mario und der Zauberer". Die Gelehrtenautobiographie: Oskar Walzel. ,Dichtung und Wahrheit" als Gegenbeispiel. Kafkas Tagebücher. Die Lyrik und das Private. ,Warum gabst du uns die tiefen Blicke?". Die Arbeiterdichtung.

Der zweite Fragenkreis: Die Probleme des Höheren

Erhebung bei Novalis. Goethes Bild des Dichters. Die Alltagssprache. Bert Brechts Gedicht ,An die Nachgeborenen“. Die Intonationen des Alltäglichen. Das Gedicht Rilkes. Lenaus Doppelsonett „Einsamkeit". Der Zerfall der Kunst. Die Probleme der Stimmungslyrik. Die geforderte Distanz: Schillers Theorie der Lyrik. Die Probleme einer ,,nihilistischen Dichtung". Kafkas ,Die Verwandlung". Der Trost des Erkennens. Ernest Hemingway: ,Ein sauberes, gut beleuchtetes Caféc". 
Sprache und isolierte Sprachkritik. Formel, Topos und Cliché. Gattungsbezeichnungen als Wertbezeichnungen: das Intrigendrama, das Konversationsstück. Die Integration der Teile: E. T. A. Hoffmann und sein ,,Meister Floh". Die Autonomie der Szene. Die epische Integration. Der Wahrheitswert im Ganzen des Kunstwerks. „,Geredet" und „,Gestaltet". Die Begriffe der klassischen Ästhetik: Stilbruch und Stimmigkeit. Hermann von Gilm: „Allerseelen". Heinrich von Kleist: ,,Das Erdbeben in Chili“. Stiltrennung und Stilmischung. Die auseinanderbrechende Ganzheit: Heinrich Heine. Der Kitsch. Die Tendenzliteratur.

Der vierte Fragenkreis: Die Probleme des Wahren

Die Rückwendung ins Historische: der historische Roman. Fluchtliteratur: die Optik Hermann Brochs. Goethe um 1813. Die Ballade des 19. Jahrhunderts: Emanuel Geibel. Der Barbarossa-Mythos. Heines Barbarossagestalt. Erich Auerbachs dargestellte Wirklichkeit. Die Bewußtseinsfragen. Max Frisch: moderne Lyrik. Verbrauchte Bilder: Hirt und Flöte. Der Fall Ernst Wiechert: „,Die Hirtennovelle“. Die Unwahrheit der Bewußtseinsverhältnisse. Darstellungen menschlichen Leides. Georg Büchner: ,Dantons Tod“. Der Fall Weinheber. Der Formenkult. Tragik und Form. Die Einheit des Künstlerischen und des Menschlichen. Ein Brief Theodor Fontanes.

Max Webers Kunstauffassung. Wladimir Weidlé: ,,Die Sterblichkeit der Musen". Die Enthumanisierung der Kunst. Karl May: Sentimentalität und Grausamkeit. Ernst Jünger: "Strahlungen". Sachlichkeit und Menschlichkeit. Reinhard Baumgart: ,Unmenschlichkeit beschreiben". Paul Celan: ,Todesfuge“.

\section{SCHLUSSBEMERKUNG 181}

Der Gesprächscharakter des Themas. Der rationale Sinn der Wissenschaft und das Irrationale. Revision der Begriffe. Überprüfung der Voraussetzungen. Methodisches Bewußtsein. Gottfried Hermann. Das Spannungsgefüge der Wissenschaft. Bewahrung und Erneuerung. Ein Wort von Ranke. 


\section{VORWORT}

Die vorliegende Schrift geht auf einen Vortrag zurück, der am 9.Juli 1964 in einer wesentlich gekürzten Fassung, aber unter demselben Titel vor den Studentengemeinden beider Konfessionen an der Universität München gehalten wurde. Von der Veranstaltung her war nicht an ein eng begrenztes Fachproblem gedacht, für das sich ausschließlich Fachgenossen interessieren; und von einer wissenschaftlichen Untersuchung im üblichen Sinn kann wohl auch jetzt nicht die Rede sein. Es werden keine abschließenden Forschungsergebnisse geboten, keine Lehre, die man lernt; denn es gibt im Bereich der Literaturwissenschaft und der Literaturkritik wenige Themen, die sich so wenig einem späteren Handbuch empfehlen wie die Probleme der literarischen Wertung. Die Beweiskraft einer geisteswissenschaftlichen Untersuchung, einer noch so sorgfältigen Interpretation ist ohnehin begrenzt, wenn es sie gibt. Sie ist es in der Frage der Wertungsprobleme erst recht. Dennoch ist die gewünschte Evidenz kein nebensächlicher Wunsch. Auch im vorliegenden Fall ist sie es nicht. Der eigentlich wissenschaftliche Sinn wird im Gegenteil gerade darin gesucht: im Versuch, die Übereinkunft so weit zu fassen wie möglich. Es sei die Natur der Humanität, hat Hegel in der »Phänomenologie des Geistes « bemerkt, auf die Übereinkunft mit anderen zu dringen. Solcher Übereinkunft das Wort zu reden bei einem Thema, das die Geister scheidet, setzt Bereitschaft zum Gespräch voraus. Sie läuft der Wissenschaft nicht zuwider, sondern erweist sich zunehmend als ihr zeitgemäßer Sinn. Dem Verfasser dieser Schrift ist die Offenheit des wissenschaftlichen Gesprächs seit langem wichtiger als die Abgeschlossenheit einer wissenschaftlichen Lehre, und vorzüglich als ein solcher Gesprächsbeitrag sind die hier dargebotenen Überlegungen ge- 
meint. Wenn über den Sinn eines solchen, durchaus vorläufigen Gesprächsbeitrages hinaus noch von einem Nebensinn zu sprechen wäre, so ist es dieser, daß bei einem Thema wie dem unseren vor Hoffnungen $\mathrm{zu}$ warnen ist, die unter keinen Umständen erfüllt werden dürfen. Auch aus solchen Gründen versteht sich die Darbietungsform ohne alle registerartigen Verzeichnisse. Wie der Vortrag ist die daraus hervorgegangene Studie als ein Ganzes gemeint. Was sie anderen Schriften verdankt, ist in den Anmerkungen gesagt.

München, im April 1965

Walter Müller-Seidel 


\section{Zur zWeiten Auflage}

Von der Beseitigung der Druckfehler abgesehen, erscheint die zweite Auflage im Text wie in den Anmerkungen unverändert. Gegenüber einem solchen Verfahren sind zahlreiche Einwände denkbar. Gewiß nicht der nebensächlichste ist der, daß wir uns im Umgang mit Literatur einer veränderten Situation gegenübersehen, an die man noch vor einigen Jahren kaum zu denken wagte. Was alles bis dahin selbstverständlich schien, ist dieses Selbstverständliche nicht mehr. Es wird mancherlei auf eine oft radikale Art in Frage gestellt; auch von jenen, die sich hauptsächlich und hauptberuflich damit befassen. Auch Kunst und Literatur werden zur Rechenschaft gezogen und haben Auskunft zu geben, warum es sie noch gibt. Man geht nicht immer glimpflich mit ihnen um, und die ,hohe" Literatur wird dabei nicht verschont. Aber muß man sich durch Vorgänge beunruhigen und beirren lassen, die vielleicht schon morgen der Vergangenheit angehören? In der Tat hat man allen Grund zu müssen; denn es sind nicht periphere Probleme, die nun schon seit geraumer Zeit die Diskussion beleben. Sie mit Indifferenz und Neutralität zu behandeln, käme einer Wirklichkeitsflucht gleich. Wir sind zur Stellungnahme gebeten und gehalten, viele Fragen neu zu überdenken. Ginge es hier um die Mitteilung unveränderlicher Fakten, so könnte man sich getrost alle zusätzlichen Erläuterungen ersparen. Mit einem Buch über Probleme derWertung und seiner Wiedervorlage verhält es sich ein wenig anders. Von gesicherten Ergebnissen kann da nicht entfernt die Rede sein. Die Problemlage wandelt sich rasch. Nichts läge daher näher, als der veränderten Situation in Form einer entsprechend veränderten Auflage Rechnung $z u$ tragen.

Es ist kein Ausweichen, wenn wir es für diesmal unterlassen. 
Daß sich der Verfasser noch in allen Punkten dort befindet, wo er vor einigen Jahren stand, erwarte man nicht. Es ist zu viel in Bewegung geraten, als daß es erlaubt wäre, in der Welt von Gestern zu denken. Aber was sich im Wandel der Dinge als echter Fortschritt erweisen könnte, ist noch keineswegs erwiesen. In dieser Hinsicht befinden sich die sogenannten Geisteswissenschaften gegenüber den Naturwissenschaften in einer etwas anderen Lage. Deren Fortschritte sind als neue Entdeckungen weithin beweisbar. Für die Deutung von Texten oder für die Darstellung historischer Zusammenhänge gelten andere „Regeln“. Hier ist das jeweils Neue mit dem Neuen einer fortschreitenden Wissenschaft nicht immer identisch. Nicht alles ist von der Zeitlage her bedingt. Vieles auch ist modisch ,bedingt". Zwischen Modenwandel und Methodenwandel gilt es daher zu unterscheiden; und diese Unterscheidung ist in mehrfacher Hinsicht erschwert. Es ist noch nicht recht abzusehen, wohin die Fahrt geht. Noch weniger ist abzusehen, welcher Kursbücher man sich hierzu bedienen soll. In solchen Situationen sieht man sich vor Übereilungen gewarnt.

Wenn aber vieles noch offen bleibt, weil es sich im Fluß befindet, so sind doch vielleicht schon jetzt einige Fortschritte der Literaturwissenschaft deutlicher zu bezeichnen, die dem Buch, wie ich hoffe, zugute gekommen sind. Darüber ein Wort zu sagen, ist schon deshalb angezeigt, weil man in Zeiten allgemeinen Umbruchs wie den unseren gern nur noch gelten läßt, was sich durch den Reiz der Neuheit empfiehlt. Der Zusammenhang mit der Tradition ist dann stärker bedroht als sonst. Auf ihn aber bleibt jede Wissenschaft angewiesen. Zwar sind ihre Resultate nichts ein für allemal Gültiges. Alle Forschung, sagt Max Weber, will überholt und überboten sein. Gleichwohl sind die Fortschritte einer Wissenschaft irreversibel: man geht nicht hinter die Positionen zurück, die einmal erreicht worden sind. Man denkt von ihnen her weiter. Das ist im Bereich der Literaturwissenschaft in den zurückliegenden Jahren auch auf vielfache Weise geschehen. Die gewiß folgenreichste Erweiterung unseres Blickfeldes ist durch die Einbeziehung literatursoziologischer Fragestellungen erfolgt. Auch die marxistische Literaturwissenschaft - in dem, was sie ist, 
wie in dem, was sie sein könnte - hat an Interesse gewonnen. Auf die Probleme der literarischen Wertung sieht man sich in solchen Gebietserweiterungen ganz unmittelbar verwiesen, wie sich am Beispiel der Trivialliteratur zeigt. Ihre Erschließung haben Soziologen, Volkskundler und Literarhistoriker gemeinsam vorangetrieben. Auch gewisse Aufwertungen dieses Schrifttums hat es hier und da gegeben; und in der Tat wäre mit einer summarischen Verurteilung nichts getan. Auch diese von der Wissenschaft zumeist verachteten Gebiete haben ein Anrecht darauf, daß man sie, wenigstens eine Weile, aus ihren eigenen Voraussetzungen versteht, damit man durchschaut, was da gemacht wird. Aber auch nur für eine Weile sind solche Tugenden des Verstehens zu üben. Von der Verpflichtung, das Minderwertige einer solchen Literatur zu erkennen, sieht man sich nicht dispensiert. Genauer noch sind bestimmte Einstellungen ihr gegenüber unerläßlich, und zwar von vornherein. Man muß wissen, welche Einstellung gegenüber diesem oder jenem Werk jeweils angemessen ist; man muß von vornherein wissen, womit man es zu tun hat. Hohe wie niedere Literatur - das hat die Erforschung der Trivialliteratur bestätigt - setzen noch vor jedem Umgang mit ihr eine Entscheidung über die Art und Weise dieses Umgangs voraus. Sie setzen mit anderen Worten noch vor jeder Interpretation eine Wertung voraus, weil man Trivialliteratur nicht in gleicher Weise interpretieren kann, wie man beispielsweise Goethes »Wahlverwandtschaften « interpretiert. Es sind jeweils andere Fragestellungen, die wir herantragen.

Der vielberufene Begriff der Autonomie steht damit zur Diskussion. Er bezieht sich auf die Literatur wie auf die Literaturwissenschaft gleichermaßen. Nichts ist gewonnen, wenn man ihn als unveränderliches Dogma in die Debatte wirft; so wenig es angebracht ist, seine Verdächtigung mit dogmatischem Eifer zu verfechten. Denn etwas „Erreichtes“, hinter das man nicht zurückgehen sollte, ist diese Autonomie nun doch. Die Anerkennung bestimmter Eigenrechte, die damit gegeben sind, hat Goethe in einem Brief an den Grafen Reinhard vorweggenommen: „Das Gedichtete behauptet sein Recht wie das Geschehene...", heißt es dort. Auf der Anerkennung solcher Eigenrechte beruht die 
neuere Literaturwissenschaft in der Eigenart ihrer Praxis wie ihrer Methoden. Das gilt für die Trivialliteratur nicht in gleicher Weise wie für die Gegenstände der , hohen“ Literatur. In diesem Punkt sind Literaturwissenschaft und Literatursoziologie nicht gänzlich dasselbe. Differenzierungen sind in jedem Fall unerläßlich. Es kann sich nicht darum handeln, im Verständnis dieser Autonomie Literatur und Gesellschaft zu trennen. Literatur ist zwar eine eigene Welt in der Welt. Aber Außerdichterisches wird in zahlreichen Formen in sie ,umgesetzt". Nicht nur Gesellschaftliches ist damit gemeint, sondern Bewußtseinsinhalte und Bewußtseinsformen der verschiedensten Art. Auch die Wege der Naturwissenschaft bleiben nicht ohne Einflu $\beta$ auf die Entwicklung einer Literatur. Auch deren, vielleicht popularisierte Resultate setzen sich in Literatur um, die uns im künstlerisch Geleisteten solcher Umsetzungen überzeugt oder auch nicht überzeugt. Im Bewußtsein des Schriftstellers oder seiner Zeit geht diese Umsetzung von der außerliterarischen Wirklichkeit in die „Wirklichkeit" der Literatur vor sich. Auf die gelejstete Umsetzung aber kommt es an, und die Wertung selbst wird von ihr wesentlich bestimmt. Es kann uns daher wenig an einem Begriff der Autonomie gelegen sein, der das ,,sprachliche Kunstwerk" als etwas ausschließlich Ästhetisches isoliert; wie uns zum andern nicht damit gedient ist, daß man diese Autonomie zugunsten einer totalen „Vergesellschaftung" alles Literarischen beseitigt. Worum es geht, sind Funktionen der Vermittlung in sprachlicher Form. Jede Literarurwissenschaft, die darauf Wert legt, von heute zu sein, hat sich um solche Theorien der Vermittlung zu kümmern. Auch die marxistische Literaturwissenschaft hat hierzu allen Grund. Mit dem Begriff der „Widerspiegelung“, wo man noch mit ihm operieren sollte, wird mehr verstellt als erhellt.

Es liegt am Gegenstand, daß man sich auf Methodenreflexionen verwiesen sieht, wie man heute zu sagen pflegt. Wertungsfragen sind Grundsatzfragen. Solche Auffassungen werden heute kaum noch bestritten. Damit ist ein zweites Moment im nicht umkehrbaren Proze $ß$ des wissenschaftlichen Fortschritts gegeben: das Interesse für Methoden und Theorien aller Art. Oft ist die Höhe der Reflexionsstufe bemerkenswert, auf der man 
diskutiert. Eine bloß musisch orientierte und theorieferne Interpretationskunst gilt nicht mehr viel. Die Literaturwissenschaft der letzten Jahre ist zunehmend reflexionsfreudig geworden. Dabei hat die Methodendiskussion bisweilen ein solches Ausma $\beta$ erreicht, da $B$ sich gewisse Bedenken regen : das Bedenken vor allem, daß man solche Reflexionen als die Sache im eigentlichen Sinn betreibt - aber ohne die Sache selbst.

In der Theologie, in den Philologien wie in allen historischen Wissenschaften ist die Hermeneutik seit je der unverzichtbare Bestandteil im Ganzen dieser Grundlagen und Grundsatzfragen gewesen. Wenigstens seit Schleiermacher war es der Fall, an dem sich auch Dilthey orientierte, dem die ältere Literaturwissenschaft das meiste verdankt. Sofern sie dabei traditionsgemä $\beta$ allen Fragen der Wertung gegenüber Entsagung übte, lag diesem Verhalten ein unverkennbarer Widerspruch zugrunde. Diese Literaturgeschichte, die vornehmlich als Geistesgeschichte betrieben wurde, hat die Hermeneutik ausschließlich als eine solche des Verstehens übernommen und dabei verkannt, wie sehr sieim Verstehen die Wertskala einer bestimmten Ästhetik mit übernahm. Deren historisch bedingte Gültigkeit hat sie nur selten erörtert. Mit einem oft unbegrenzt anmutenden Verstehen im Geltungsbereich dieser Ästhetik schien ein historischer Wertrelativismus gegeben zu sein, der in Wirklichkeit nur verdeckte, daß man gegenüber anderen Erscheinungen des literarischen Lebens außerhalb dieses Bereiches weniger verständnisbereit war. Das entsprach, bei Lichte besehen, nicht einmal der Tradition der historischen Schule. Dennoch ist mit dem heute zu fordernden Verzicht auf solche Positionen als $\mathrm{Maß}$ und Norm ein Rückfall in den Relativismus dieser Schule zu vermeiden. Auch hier muß es darauf ankommen, daß man nicht hinter die erreichte Stufe zurückgeht, und der Historismus ist eine Stufe dieser Art. Von sich aus wirken literarische Wertungen einem solchen Relativismus ohnehin entgegen. Sie setzen dem Verstehen gewisse Grenzen und ermöglichen damit die Kritik, die sich ihrerseits einem normativen Denken annähert. Das Spannungsverhältnis von Hermeneutik und Wertung, von Relativität und Norm, das dadurch entsteht, ergibt einen Zirkel, der genauer zu beschreiben wäre. Aber der hermeneu- 
tische Zirkel im alten Sinne ist es nicht. Indem sich die neuere Hermeneutik auf eine Theorie der Wertung einläßt und sich dabei auf das Autorenbewußtsein nicht mehr beschränkt, sind die Grenzen ihres bisherigen Geltungsbereiches beträchtlich erweitert. Die Wertungen erhálten innerhalb dieses Geltungsbereiches ihre relative Relevanz, und die hermeneutischen Voraussetzungen und Bedingungen lassen uns dabei an eine Art Modell denken, an einen Bereich von bestimmter zeitlicher Dauer, der uns gewiß nicht ästhetische Urteile von uneingeschränkter Objektivität erwarten läßt, aber die Beliebigkeit subjektiven Meinens dennoch einschränkt. Für das Modellhafte solcher Zeitbezüge gebraucht man im Kreis des Münchener Altgermanisten Hugo Kuhn den Begriff der Situation, der sich nach der Seite des Geschichtlichen wie nach der Seite der ungeschichtlichen Anthropologie erläutern läßt. Über einige weiterführende Fragen dieser Art habe ich in einem Beitrag gehandelt, der vor kurzem in der Zeitschrift /Der Deutschunterricht، erschienen ist (»Wertung und Wissenschaft im Umgang mit Literatur «, in : Der Deutschunterricht, 1969, Heft 3).

Literarische Wertung ist Urteilsbildung im Umgang mit Literatur. Aber oft sind es die Vorurteile, die uns den Blick für die tatsächlichen Gegebenheiten verstellen. Im positiven Sinn können sie als Vorbedingung geschichtlichen Verstehens aufgefaßt werden. Als solche hat sie Hans-Georg Gadamer in seinem Buch »Wahrheit und Methode« zu Recht rehabilitiert. In negativer Hinsicht stellen sie Verhärtungen und Verfestigungen dar, die in Ideologie übergehen. Was sie in solcher Beschaffenheit anzurichten und anzustiften in der Lage sind, haben Alexander und Margarete Mitscherlich unlängst beschrieben. In dem Buch »Die Unfähigkeit zu trauern« führen sie über das Phänomen des Vorurteils aus: „Es drängt sich uns mit Selbstverständlichkeit auf und schläfert oder schüchtert unser kritisches Ich ein. Wir errichten mit Annahme und Übernahme von Einstellungen, die von Vorurteilen bestimmt sind, ein rigides System in uns selbst, meist ohne es zu wissen ... Wir erliegen hier einem ,Reflexionsblock', einer Reflexionslähmung, können vorurteilsbesetzten Komplexen der Realität gegenüber plötzlich nicht mehr nach- 
denken, reflektieren, uns abwägend verhalten, sondern es erscheint uns ein Stück Welt mit Einzelcharakter, als so und nicht anders, als so selbstverständlich, da $\beta$ es sich gar nicht lohnt, eine Frage darauf zu verschwenden, Zweifel kommen uns nicht in den Sinn..." Vorurteile sind stets auch einseitige Urteile und in solcher Einseitigkeit wissenschaftlichem Denken abträglich. Nichts eigentlich sollte einer Wissenschaft mehr zur Ehre gereichen als Vorurteilsforschung an sich selbst zu treiben, und das schließt ein, daß man Theorie wie Praxis seiner wissenschaftlichen Tätigkeit immer erneut auf ihre unaufgehellten Voraussetzungen befragt und überprüft.

In der Geschichte der deutschen Germanistik ist die klassische Ästhetik als das alleingültige Maß der Dinge lange Zeit und viel zu lange ein solcherart unaufgehelltes Vorurteil gewesen. Daß sie diese normative Funktion heute in nahezu allen Bereichen des literarischen Lebens eingebüßt hat, steht außer Frage. Die klassische Ästhetik ist ein Wert unter anderen; und auf eine Vielzahl gleichberechtigter und verwandter "Werte" haben wir uns zu richten. Alle ewigen und unwandelbaren Gesetze lassen wir lieber aus dem Spiel. Soweit, darf man annehmen, ist die Einsicht allgemein geworden; und darin abermals darf man ein Moment des Fortschritts sehen, den rückgängig zu machen wir uns hüten wollen. Aber zu unbekümmerter Fortschrittsgläubigkeit besteht kein Grund. Es ist sehr die Frage, ob man sich überall der so völlig ungesicherten Position'bewußt ist, der wir uns damit gegenübersehen. Die Gesetzbücher gibt es ein für allemal nicht: die Kataloge mit den zuverlässig verzeichneten Wertungskriterien, die sich manche vielleicht wünschen. Es ist alles offen, wie es sich freilich für eine Wissenschaft von selbst verstehen sollte, in der man gelernt hat, vorurteilsfrei zu denken. Der Verzicht auf die Sicherheit einer alles regelnden Norm schließt eine Bereitschaft ein, die sich möglicherweise noch zu bewähren hat: die Bereitschaft zur Anerkennung der schlechterdings konträren „Werte“, was immer man unter diesen verstehen will. Die somit geforderte Vielseitigkeit, die ,Mehrwertigkeit" der Phänomene, Formen und Begriffe ist das Neue einer vielleicht noch nicht durchweg durchschauten Situation, die das Geschäft der Wertung beträchtlich 
kompliziert. Das Naive wie das Sentimentalische, das Gesunde wie das Kranke, die Veränderung wie die Bewahrung, die fortdauernde Unruhe (im Hinblick auf Faust) wie die höchste Ruhe in Gott, können so oder so beurteilt werden, ohne daß uns im vorhinein die Kriterien der Wertung zur Verfügung stünden. Das Verharren - eines Schriftstellers oder einer Epoche - in einem Teil des Ganzen hat den Umschlag in sein Gegenteil zur Folge. Auch in der geschichtlichen Welt erweisen sich die Phänomene, über die wir uns ein Urteil bilden sollen, als komplementär. Das Eine korrigiert sich am Anderen: der ungestüm aufbegehrende Sturm und Drang an der nachfolgenden Besonnenheit der Klassik, die zunehmend wirklichkeitsferne Romantik am Realismus des Alltäglichen, der ihm folgt, und so fort. Jede Epoche hat die Tendenz, sich im Eigenen ihrer Anfänge zu verfestigen. Sie begünstigt in der Eigenart ihrer Formen die Einseitigkeit und die Einsinnigkeit dieser Formen, und in der Einseitigkeit die Neigung zu Ideologien aller Art. Von hieraus erweist sich auch die Parteilichkeit der Wertung als höchst bedenklich.

In den Ländern Westeuropas hat eine solche Parteilichkeit als Prinzip der Wissenschaft kaum je Bedeutung erlangt. Sie blieb auf die Theorien des Marxismus in den östlichen Ländern beschränkt. Welche Entfremdungen vom wissenschaftlichen Denkken sich damit verbinden können, hat Werner Hofmann in einer Analyse des Stalinismus aufgezeigt. ,,Als Kriterien der Aussagen“, führt er aus, ,,erschien nicht ihreWahrheit, sondern ihre Nützlichkeit oder Schädlichkeit im Sinne vorgegebener Zwecke. Und die Losung von der Parteilichkeit ward für viele Denker zur bequemen Lizenz, nicht aufgrund sorgsamer Analyse der Tatbestände schließlich Partei zu ergreifen, sondern vielmehr als Partei an die Sachverhalte selbst schon wählend und sichtend heranzutreten."Hier ist ausgesprochen, da $\beta$ der Verzicht auf Parteilichkeit nicht mit einer Standortlosigkeit verwechselt werden darf, die zu nichts verpflichtet. Für eine Sache gegebenenfalls Partei zu ergreifen, wird niemand verdacht, und solche Parteinahme kann zu Zeiten durchaus im Namen einerWissenschaft erfolgen. Dennoch gilt es, vor unguten Vermischungen und Verwechslungen in diesem Problembereich auf der Hut zu sein, weil es nur allzu leicht geschehen 
kann und tatsächlich geschieht, daß man in der Darbietung von Sachverhalten Partei ergreift, ohne durchschaut zu haben, daß man an sie schon zuvor als Partei herangetreten ist: mit festen und mit festgelegten Überzeugungen. Solche Überzeugungen und Gesinnungen können abermals die Funktion einer Norm übernehmen, an der alles und jedes gemessen wird. Hier vor allem sind seit dem Erscheinen der ersten Auflage einige Abgrenzungen nötig. Es ging noch vor wenigen Jahren weit mehr darum, eine wissenschaftlich vertretbare Wertung gegenüber der Tradition des Verstehens zu legitimieren, während heute vorzüglich darauf zu sehen ist, daß man Wertungen in dem hier vertretenen Sinn mit Parteilichkeit nicht verwechselt. Daß diese sich in ihrer einseitigen Fixierung über kurz oder lang als Ideologie enthüllt, wird sich zeigen. In diesem Zusammenhang ist eine Besorgnis nicht ganz unbegründet: die Besorgnis nämlich, es könnten sich neue Normen bilden, die uns sehr dezidierte Kriterien der Wertung zur Verfügung stellen, solche von womöglich politischer Relevanz im ausschließlichen Sinne. Neue Vorurteile der literarischen Urteilsbildung müßten die Folge sein, die man auch dann als Ideologie zu bezeichnen hat, wenn sie vorgeben, anders geartete Ideologien zu bekämpfen. Es kann nicht der geschichtliche Sinn sein, daß man die Bindung an fixierte Normen und Regeln durch eine Bindung mit entsprechenden Normen eintauscht und ersetzt. Und darauf liefe es zuletzt doch wohl hinaus : da $\beta$ wir uns erneut einem fest umrissenen Geltungsbereich gegenübersehen - diesmal einem solchen von ausschließlich politischer Relevanz -, nachdem wir uns aus jenem der alten Ästhetik glücklich herausgefunden haben. Die gewisse Bindungslosigkeit, die nichts Unverbindliches meint, ist der Fortschritt, den wir nicht verspielen sollten. Radikale Unabhängigkeit des Denkens - aber nach allen Seiten hin - ist das, was von uns gefordert wird. Auch Offenheit ist Engagement.

Die „Zwischenbemerkung", wie wir die Stellungnahme im Rahmen dieses Vorwortes bezeichnen wollen, drängt unverzüglich zur Erörterung derjenigen Fragen hin, die heute in aller Munde sind und vielleicht den eigentlichen „Herd" aller (fruchtbaren) Unruhen darstellen. Ich meine damit die Erarbeitung eines neuen 
Wissenschaftsbegriffs, der ohne Zweifel zu denjenigen Forderungen des Tages gehört, die einen Vorrang vor anderen Forderungen beanspruchen. Schon aus diesem Grund wäre es aussichtslos, sich auf die Erörterung von Problemen einzulassen, die man beim besten Willen nicht im Vorbeigehen erledigt. Es kann sich daher in unserem Zusammenhang nur um knappe Hinweise handeln, die zumal nach dieser Seite hin bestätigen, was eingangs thesenhaft gesagt war: daß sich die Dinge noch im Fluß befinden und ein zuverlässiges Urteil einstweilen erschweren. Es ist aber kein Zweifel, daß Wertungsfragen - im literarischen Bereich und andernorts - auf einen bestimmten und vermutlich nicht ganz herkömmlichen Wissenschaftsbegriff bezogen sind. Er wird zum Fluchtpunkt, auf den alles zuzulaufen scheint. Ein solcher Begriff von Wissenschaft, dessen hier abschließend wenigstens gedacht sein soll, wenn er nicht explizit beschrieben werden kann, wird sich zweifellos um das zu kümmern haben, was bei uns und in aller Welt innerhalb der naturwissenschaftlichen Forschung Wissenschaft heißt; und es bekäme den traditionellen Geisteswissenschaften sicher nicht schlecht, wenn sie nachhaltiger davon Kenntnis nehmen wollten, was alles dort gedacht wird und geschieht. Von unserem Thema selbst hätte man sich damit, so scheint es, vollends entfernt. Wertungen sind in der Naturwissenschaft unmittelbar kaum je relevant. Aber gänzlich irrelevant sind sie nicht. Wertung in der Wissenschaft ist, zu Ende gedacht, nicht ablösbar vom Wert der Wissenschaft selbst. Das kann nur heißen : vomWert, den sie für den Menschen besitzt. Fragen dieser Art sind Schriftsteller wie Literarhistoriker wie Naturforscher sich vorzulegen verpflichtet. Sie haben zuletzt ein gemeinsames Ziel, wie unterschiedlich auch die Wege sein mögen, die sie auf Grund ihres Metiers einschlagen, um dorthin zu gelangen. Jede Wertung tendiert zuletzt auf so etwas wie einen höchsten Wert hin : auf etwas jenseits solcher Tätigkeiten im bloß pragmatischen Sinn. Denn Wissenschaft um ihrer selbst willen ist so wenig der höchste Wert wie eine Kunst, die nichts anderes kennt als sich selbst, falls es eine solche je gegeben hat. WelcherWert aber könnte es sein, der uns in allem Tätigsein für die Wissenschaft auf ein Jenseits derWissenschaft und in allem Tätigsein für die Kunst auf 
ein Jenseits der Kunst verweist? Spätestens an diesem Punkt muß sich zeigen, daß wir alle Leistungen des menschlichen Geistes immer nur eine Zeit lang zu isolieren berechtigt sind und daß es mehr denn je darum gehen muß, vieles mit vielem zu verbinden. Es wird kein Zufall sein, daß es ein Schriftsteller unserer Zeit ist, der solches tut und dabei in literarischer Form einen Begriff von Wissenschaft vorwegnimmt, auf den man vermutlich zurückkommen wird, wo immer es einen neuen, unserer heutigen Welt entsprechenden Wissenschaftsbegriff zu erarbeiten gilt. Den Galilei seines Dramas läßt Brecht sagen, was wir uns gern zu eigen machen wollen: „Ich halte dafür, daß das einzige Ziel der Wissenschaft darin besteht, die Mühseligkeit der menschlichen Existenz zu erleichtern. Wenn Wissenschaftler, eingeschüchtert durch selbstsüchtige Machthaber, sich damit begnügen, Wissen um des Wissens willen aufzuhäufen, kann die Wissenschaft zum Krüppel gemacht werden, und eure neuen Maschinen mögen nur neue Drangsale bedeuten. Ihr mögt mit der Zeit alles entdecken, was es zu entdecken gibt, und euer Fortschritt wird doch nur ein Fortschreiten von der Menschheit weg sein ..." (14. Szene). Denn Fortschritte in der Wissenschaft, wie uns hier zu bedenken gegeben wird, müssen nicht in jedem Fall Fortschritte der Menschlichkeit sein. Sie sollen es erst werden. Daß es ein Schriftsteller ist, der hier so unerwartet der Wissenschaft zu Hilfe kommt, soll uns nur freuen - um der Literatur willen nicht zuletzt derentwegen wir kein schlechtes Gewissen zu haben brauchen, wenn wir an ihr weiterhin diejenige Freude haben, die sie noch immer verdient.

München, im August 1969

W. M-S 
„ICH HABE KEINE LEHRE, ABER ICH FÜHRE ein Gespräch" (Martin Buber) 